1 Hacettepe Journal of Mathematics and Statistics

$\bigcap$ Volume 47 (2) (2018), 299-316

\title{
Stability analysis of distributed order Hilfer-Prabhakar differential equations
}

\author{
M. Mashoof*, A.H. Refahi Sheikhani ${ }^{\dagger \ddagger}$ and H. Saberi Najafi ${ }^{\S}$
}

\begin{abstract}
In the current study we presented a distributed order form of HilferPrabhakar (DHP) derivative, which in special cases reduces to the existent definitions of fractional or distributed order derivatives. Moreover, we analyzed the stability of DHP differential equations, which are the generalized form of all previous distributed or fractional differential equations. The obtained results showed that sufficient conditions on asymptotic stability of these systems have been obtained through the generalized properties of Mittag-Leffler functions and the Laplace transform. Moreover, a number of conditions on stability analysis of such systems have been introduced by using a new definition of inertia of a matrix.
\end{abstract}

Keywords: Distributed order fractional derivative, Hilfer-Prabhakar derivative, Asymptotic stability.

2000 AMS Classification: 26A33; 93D20

Received : 07.10.2016 Accepted : 27.03.2017 Doi : 10.15672/HJMS.2017.470

*Department of Applied Mathematics, Faculty of Mathematical Sciences, Lahijan Branch, Islamic Azad University, Lahijan, Iran. Email: mashoof.mohammad@yahoo.com

${ }^{\dagger}$ Department of Applied Mathematics, Faculty of Mathematical Sciences, Lahijan Branch, Islamic Azad University, Lahijan, Iran., Email: ah_refahi@liau.ac.ir

${ }^{\ddagger}$ Corresponding Author.

$\S$ Department of Applied Mathematics, Faculty of Mathematical Sciences, Lahijan Branch, Islamic Azad University, Lahijan, Iran., Email: hnajafi@guilan.ac.ir 


\section{Introduction}

Differential equations and fractional differential equations are widely applied realms in physics and engineering; instances of them can be found in Lorenz and Chen's differential equations [8, 1], heat diffusion beyond Fourier law [51], Guyer-Krumhansl type heat equation [46, 47], distributed order fractional Riccati differential equation [4, 2, 10], pulsar radiation in post-Maxwellian vacuum nonlinear electrodynamics [19], and Black-Scholes equations [49]. Moreover, there are many important questions relevant to differential equations; three of the most notable are those of existence and uniqueness of solutions and their stability and asymptotic stability. In this regard, in [48, 43, 52] solutions of some important differential equations, arising in physics, have been dealt with. One of the most important differential systems is Lorenz and Chen's nonlinear systems, which arise in various fields such as physical [31], chemical [25], and ecological systems [15]. Similar to the integer order systems, its fractional order derivative has been studied by a number of researchers including [8, 3, 50], to name but a few.

The origins of the field of fractional calculus dates back to more than three centuries ago; however, the field has received attention and interest only in the past 20 years or so $[30,9,7,45]$. Fractional calculus is the generalization of calculus, in which the order of derivatives and integrals are arbitrary numbers in the form of Caputo fractional derivative, Riemann-Liouville fractional derivative, or Hilfer-Prabhakar derivative. Here, a notable aspect is that engineers and scientists have developed new models that involve fractional differential equations. These models have been applied widely in mechanics (theory of viscoelasticity and viscoplasticity), (bio-)chemistry (modelling of polymers and proteins), electrical engineering (transmission of ultrasound waves), medicine (modelling of human tissue under mechanical loads), etc.[20].

The distributed-order operators can be obtained by integrating the fractional-order calculus operators with respect to the order variable. Caputo was the first one to discuss the distributed order differential equation [17] and further developed the concept himself $[16,17]$ along with scholars such as [14]. In [10], the multi-term fractional derivative viscoelastic model has been generalized to a derivative model of distributed order by replacing the finite sums with integrals in the domain of orders. Based on this model Atanackovic analyzed the response of several systems such as the fractional distributed order oscillator [11] and the distributed order fractional wave equation [12, 13].

A definition of the distributed-order derivative is as

$$
d_{o} \cdot D_{t}^{\alpha} f(t)=\int_{0}^{1} b(\alpha)_{a^{+}} \cdot D_{t}^{\alpha} f(t) d \alpha
$$

where, the fractional derivative of integrand function is in Caputo or in Riemann-Liouville form. $[8,39,5]$ investigated the stability analysis of distributed order differential equations with respect to the nonnegative density function; in their study the fractional derivative of distributed order was based on the Caputo's definitions. In the current paper, we will deal with a new definition of distributed order derivative with respect to Hilfer-Prabhakar derivative [23, 36, 35] that is called the distributed order HilferPrabhakar derivative. We will also show that the distributed order Hilfer-Prabhakar derivative can be reduced to the Caputo or Riemann-Liouville fractional derivative, Hilfer or Hilfer-Prabhakar derivative, and the distributed-order derivative in Caputo or Riemann-Liouville form. Regarding these trends of research, [40] studied stability analysis of Hilfer fractional differential systems.

This paper is organized as follows. In section 2, we review some basic definitions of fractional and distributed order fractional derivative operators. Our new definition of distributed order fractional derivative and its properties will be presented in this section. Section 3 deals with the main theorems for checking the stability analysis of distributed 
order Hilfer-Prabhakar derivative systems. Moreover, we will see that this system can be reduced to a system with previous fractional or distributed order fractional derivatives. In section 4 we illustrate our new theorems and definitions by three examples. Finally, conclusions are presented in section 5 .

\section{Preliminaries}

Here and in this part of the study, we present our new definition of the distributed order derivative together with some basic definitions related to fractional calculus. Moreover, we will check whether the other fractional derivatives are expressible in terms of our proposed definition. Some concepts and techniques related to Laplace transforms are presented as well.

The Mittag-Leffler function and its generalizations, which has been introduced and studied by [33, 34], [44] and [28], have been presented as the solution of fractional order differential equations and fractional order integral equations. Due to its direct applications in fields such as physics, biology, engineering, and applied sciences, its importance has been emphasized during the last 15 years or so [6, 24].

2.1. Definition. The Mittag-Leffler function has been defined by the series representation as

$$
E_{\beta}(z)=\sum_{n=0}^{\infty} \frac{1}{\Gamma(\beta n+1)} z^{n}, \quad \beta>0, z \in \mathbb{C},
$$

and its generalization by the following form

$$
E_{\beta, \gamma}(z)=\sum_{n=0}^{\infty} \frac{1}{\Gamma(\beta n+\gamma)} z^{n}, \quad \beta>0, \gamma>0, z \in \mathbb{C},
$$

where $\mathbb{C}$ is the set of complex numbers.

Applying the series representation, a generalization of (2.2) has been introduced by [37] as:

$$
E_{\beta, \gamma}^{\delta}(z)=\sum_{n=0}^{\infty} \frac{(\delta)_{n}}{\Gamma(\beta n+\gamma) n !} z^{n},
$$

where $\beta, \gamma, \delta \in \mathbb{C}$, with $\Re(\beta), \Re(\gamma)>0$ and $(\delta)_{n}$ is the Pochhammers symbol defined as

If we define $e_{\rho, \mu \omega}^{\gamma}$ as

$$
(\delta)_{n}=\delta(\delta+1) \cdots(\delta+n-1)=\frac{\Gamma(\delta+k)}{\Gamma(\delta)}, \quad(\delta)_{0}=1 .
$$

$$
e_{\rho, \mu \omega}^{\gamma}(t)=t^{\mu-1} E_{\rho, \mu}^{\gamma}\left(\omega t^{\rho}\right), \quad t \in \mathbb{R}, \rho, \mu, \omega, \gamma \in \mathbb{C}, \Re(\rho), \Re(\mu)>0,
$$

then we have the following lemma,

2.2. Lemma. The Laplace transform of $e_{\rho, \mu \omega}^{\gamma}(t)$ is given by

$$
\mathcal{L}\left[e_{\rho, \mu \omega}^{\gamma}(t)\right](s)=s^{-\mu}\left(1-\omega s^{-\rho}\right)^{-\gamma} .
$$

2.3. Definition. The Riemann-Liouville fractional integral of order $\alpha>0$ for an absolutely integrable function $f(t)$ on $t>a$ will be as

$$
{ }_{a^{+}} I_{t}^{\alpha}(f(t))=\frac{1}{\Gamma(\alpha)} \int_{a}^{t}(t-\tau)^{\alpha-1} f(\tau) d \tau=\left(f * k_{\alpha}\right)(t), \quad \alpha>0
$$

in which $\Gamma$ is the Gamma function, $k_{\alpha}(t)=t^{\alpha-1} / \Gamma(\alpha)$, and $f * k_{\alpha}$ is the convolution of $f$ and $k_{\alpha}$, and ${ }_{0^{+}} I_{t}^{0}(f(t))=f(t)$. 
Let $f \in L^{1}[a, b],-\infty \leq a<t<b \leq \infty$ and $f * k_{m-\alpha} \in W^{m, 1}[a, b], m=\lceil\alpha\rceil, \alpha>0$, where $W^{m, 1}[a, b]$ is the Sobolev space defined as

$$
W^{m, 1}[a, b]=\left\{f \in L^{1}[a, b]: \frac{d^{m}}{d t^{m}} f \in L^{1}[a, b]\right\} .
$$

The Riemann - Liouville derivative of order $\alpha>0$ is defined as

$$
{ }_{a^{+}}^{R L} D_{t}^{\alpha} f(t)=\frac{d^{m}}{d t^{m}}{ }_{a^{+}} I_{t}^{m-\alpha}=\frac{1}{\Gamma(m-\alpha)} \frac{d^{m}}{d t^{m}} \int_{a}^{t}(t-s)^{m-1-\alpha} f(s) d s .
$$

Now let $\alpha>0, m=\lceil\alpha\rceil$ and $f \in A C^{m}[a, b]$; the Caputo derivative of order $\alpha>0$ is defined as

$$
{ }_{a^{+}}^{C} D_{t}^{\alpha} f(t)={ }_{a^{+}} I_{t}^{m-\alpha} \frac{d^{m}}{d t^{m}} f(t)=\int_{a}^{t}(t-s)^{m-1-\alpha} \frac{d^{m}}{d s^{m}} f(s) d s,
$$

where $A C^{m}[a, b]=\left\{f:[a, b] \rightarrow \mathbb{R}: \frac{d^{m-1}}{d t^{m-1}} f(t) \in A C[a, b]\right\}$, and $A C[a, b]$ is the space, including absolutely continuous functions.

The Laplace transform of the Caputo fractional derivative satisfies

$$
\mathcal{L}\left({ }_{a^{+}}^{C} D_{t}^{\alpha} f(t)\right)=s^{\alpha} \mathcal{L}(f(t))-\sum_{k=0}^{m-1} f^{(k)}\left(0^{+}\right) s^{\alpha-1-k} .
$$

Recently, Hilfer has introduced a generalized form of the Riemann-Liouville fractional derivative [26]. In short, Hilfer fractional derivative is an interpolation between the Riemann-Liouville and Caputo fractional derivatives with applications in fractional evolutions equations [27].

2.4. Definition. (Hilfer derivative). Let $\mu \in(0,1), \nu \in[0,1], f \in L^{1}[a, b],-\infty \leq a<$ $t<b \leq \infty, f * k_{(1-\nu)(1-\mu)} \in A C^{1}[a, b]$, the Hilfer derivative is defined as

$$
{ }_{a^{+}} D_{t}^{\mu, \nu} f(t)=\left({ }_{a}+I_{t}^{\nu(1-\mu)} \frac{d}{d t}\left({ }_{a^{+}} I_{t}^{(1-\nu)(1-\mu)} f\right)\right)(t) .
$$

For $\nu=0$, the Hilfer derivative coincides with the Riemann-Liouville derivative, and for $\nu=1$ it does the same with the Caputo derivative. Hereafter and without loss of generality we set $a=0$.

The Prabhakar integral [29] is obtained by modifying the Riemann-Liouville integral operator by extending its kernel with a three-parameter Mittag-Leffler function, a function which extends the well-known two-parameter Mittag-Leffler function.

2.5. Definition. (Prabhakar integral). Let $f \in L^{1}[0, b], 0<t<b \leq \infty$; the Prabhakar integral can be written as

$$
\mathbf{E}_{\rho, \mu, \omega, 0^{+}}^{\gamma} f(t)=\int_{0}^{t}(t-y)^{\mu-1} E_{\rho, \mu}^{\gamma}\left(\omega(t-y)^{\rho}\right) f(y) d y=\left(f * e_{\rho, \mu, \omega}\right)(t),
$$

where $\rho, \mu, \omega, \gamma \in \mathbb{C}$, with $\Re(\rho), \Re(\mu)>0$.

Similar to the classical fractional operators, a related differential operator can be defined as follows.

2.6. Definition. (Prabhakar derivative).Let $f \in L^{1}[0, b], 0<t<b \leq \infty, f * e_{\rho, m-\mu \omega}^{-\gamma}(\cdot) \in$ $W^{m, 1}[0, b]$ and $m=\lceil\mu\rceil$; the Prabhakar derivative will be defined as

$$
\mathbf{D}_{\rho, \mu, \omega, 0^{+}}^{\gamma} f(t)=\frac{d^{m}}{d t^{m}} \mathbf{E}_{\rho, m-\mu \omega, 0^{+}}^{-\gamma} f(t),
$$

where $\rho, \mu, \omega, \gamma \in \mathbb{C}$, with $\Re(\rho), \Re(\mu)>0$. 
The Riemann-Liouville integrals in (2.6) can be expressed in terms of Prabhakar integrals as

$$
0^{+} I_{t}^{m-\mu+\theta} f(t)=\mathbf{E}_{\rho, m-(\mu+\theta), \omega, 0^{+}}^{0} f(t)
$$

therefore,

$$
\mathbf{D}_{\rho, \mu, \omega, 0^{+}}^{\gamma} f(t)={ }_{0^{+}} D_{t}^{\mu+\theta} \mathbf{E}_{\rho, \theta, \omega, 0^{+}}^{-\gamma} f(t) \quad \theta \in \mathbb{C}, \Re(\theta)>0 .
$$

For Prabhakar integral we have (see [29], Theorem 8)

$$
\mathbf{E}_{\rho, \mu, \omega, 0^{+}}^{\gamma} \mathbf{E}_{\rho, \nu, \omega, 0^{+}}^{\delta} f(t)=\mathbf{E}_{\rho, \mu+\nu, \omega, 0^{+}}^{\gamma+\delta} f(t) .
$$

As expected, the inverse operator (2.13) of the Prabhakar integral generalizes the RiemannLiouville derivative. It's regularized Caputo counterpart is given for functions $f \in$ $A C^{m}[0, b], 0<t<b \leq \infty$ by

$$
\begin{aligned}
& { }^{C} \mathbf{D}_{\rho, \mu, \omega, 0^{+}}^{\gamma} f(t)=\mathbf{E}_{\rho, m-\mu, \omega, 0^{+}}^{-\gamma} \frac{d^{m}}{d t^{m}} f(t) \\
= & \mathbf{D}_{\rho, \mu, \omega, 0^{+}}^{\gamma} f(t)-\sum_{k=0}^{m-1} t^{k-\mu} E_{\rho, k-\mu+1}^{-\gamma}\left(\omega t^{\rho}\right) f^{(k)}\left(0^{+}\right) .
\end{aligned}
$$

Hilfer-Prabhakar derivative is similar to the Hilfer derivative but based on Prabhakar operators. This Hilfer-Prabhakar derivative interpolates the two operators (2.13) and (2.17); let us give the following definition [36]

2.7. Definition. (Hilfer-Prabhakar derivative). Let $\mu \in(0,1), \nu \in[0,1]$ and let $f \in$ $L^{1}[a, b], 0<t<b \leq \infty, f * e_{\rho,(1-\nu)(1-\mu), \omega}^{-\gamma(1-\nu)}(\cdot) \in A C^{1}[0, b]$; the Hilfer-Prabhakar derivative is defined by

$$
\mathcal{D}_{\rho, \omega, 0^{+}}^{\gamma, \mu, \nu} f(t)=\left(\mathbf{E}_{\rho, \nu(1-\mu), \omega, 0^{+}}^{-\gamma \nu} \frac{d}{d t}\left(\left(\mathbf{E}_{\rho,(1-\nu)(1-\mu), \omega, 0^{+}}^{-\gamma(1-\nu)}\right) f\right)\right)(t),
$$

where $\gamma, \omega \in \mathbb{R}, \rho>0$ and $\mathbf{E}_{\rho, 0, \omega, 0^{+}}^{0} f=f$.

We have observed that (2.18) reduces to the Hilfer derivative for $\gamma=0$. Moreover, for $\nu=1$ and $\nu=0$, it coincides with (2.16) and (2.14) respectively (note $m=1$ ).

2.8. Lemma. The Laplace transform of (2.17) is given by[22]

$$
\begin{gathered}
\mathcal{L}\left(\mathcal{D}_{\rho, \omega, 0^{+}}^{\gamma, \mu, \nu} f(t)\right)=s^{\mu}\left(1-\omega s^{-\rho}\right)^{\gamma} \mathcal{L}(f)(s)- \\
-s^{-\nu(1-\mu)}\left(1-\omega s^{-\rho}\right)^{\gamma \nu}\left[\boldsymbol{E}_{\rho,(1-\nu)(1-\mu), \omega, 0^{+}}^{-\gamma(1-\nu)} f(t)\right]_{t=0^{+}} .
\end{gathered}
$$

2.9. Definition. (Distributed order derivatives). The fractional derivatives with distributed order in the Caputo or Riemann-Liouville formulations with respect to orderdensity function $b(\alpha) \geq 0$ are defined as

$$
{ }_{d o} \cdot D_{t}^{\alpha} f(t)=\int_{m-1}^{m} b(\alpha)_{a}+D_{t}^{\alpha} f(t) d \alpha
$$

where $m-1<\alpha \leq m$ and ${ }_{a^{+}} D_{t}^{\alpha}$ is the Caputo or Riemann-Liouville derivative.

For the Laplace transform of the Caputo fractional derivative of distributed order the following equation holds

$$
\mathcal{L}\left(\underset{d o}{C} D_{t}^{\alpha} f(t)\right)=B(s) \mathcal{L}(f(t))-\sum_{k=0}^{m-1} \frac{1}{s^{k+1}} B(s) f^{(k)}\left(0^{+}\right),
$$

where

$$
B(s)=\int_{m-1}^{m} b(\alpha) s^{\alpha} d \alpha
$$


Now and in this section of this study we are going to present a new definition for the distributed order derivative, by which the derivatives referred to in the previous part can be re-expressed in a new form.

2.10. Definition. Let $\mathcal{D}_{\rho, \omega, 0^{+}}^{\gamma, \mu, \nu} f(t)$ be the Hilfer-Prabhakar derivative of function $f$; the distributed order Hilfer-Prabhakar derivative with respect to the order-density function $b(\mu, \nu) \geq 0$ is defined as

$$
{ }_{d o}^{H P} \mathcal{D}_{\rho, \omega, 0^{+}}^{\gamma, \mu, \nu} f(t)=\int_{0}^{1} \int_{0}^{1} b(\mu, \nu) \mathcal{D}_{\rho, \omega, 0^{+}}^{\gamma, \mu, \nu} f(t) d \mu d \nu .
$$

2.11. Remark. Consider the density function $b(\mu, \nu)$ in the following cases

Case1. $b(\mu, \nu)=\delta\left(\mu-\mu_{0}\right) \delta\left(\nu-\nu_{0}\right)$,

Case2. $b(\mu, \nu)=b(\mu) \delta\left(\nu-\nu_{0}\right)$, where $\delta($.$) is the Dirac delta function.$

Based on the above formulations, it would be clear that in case one we have

$$
{ }_{d o}^{H P} \mathcal{D}_{\rho, \omega, 0^{+}}^{\gamma, \mu, \nu} f(t)=\mathcal{D}_{\rho, \omega, 0^{+}}^{\gamma, \mu_{0}, \nu_{0}} f(t)
$$

and, if in addition $\nu_{0}=0$ and $\omega=0$, then

$$
{ }_{d o}^{H P} \mathcal{D}_{\rho, \omega, 0^{+}}^{\gamma, \mu, \nu} f(t)={ }_{0^{+}}^{R L} D_{t}^{\mu_{0}} f(t),
$$

and, if $\nu_{0}=1$ and $\omega=0$, then

$$
{ }_{d o}^{H P} \mathcal{D}_{\rho, \omega, 0^{+}}^{\gamma, \mu, \nu} f(t)={ }_{0^{+}}^{C} D_{t}^{\mu_{0}} f(t) .
$$

Moreover, in case 2 for $\nu_{0}=1$ and $\omega=0,{ }_{d o}^{H P} \mathcal{D}_{\rho, \omega, 0^{+}}^{\gamma, \mu, \nu}$ reduces to the fractional derivative with distributed order in the Caputo form, and for $\nu_{0}=0$ and $\omega=0$ they reduce to the fractional derivative with distributed order in the Riemann-Liouville form with respect to order-density function $b(\mu)$.

Remark 2.11 shows that our new definition for distributed order fractional derivative can be reduced to all previous definitions in this section.

In the next section we consider the linear differential system with distributed order HilferPrabhakar derivative in two cases. We also illustrate some conditions of their stability. The important point not to forget her is that we can see that this system can be reduced to the system referred to in $[41,32,38]$.

\section{A Stability Analysis of Distributed Order Hilfer-Prabhakar Differential Systems}

In this section, we first discuss the stability analysis of the linear distributed order Hilfer-Prabhakar differential system as

$$
\begin{aligned}
{ }_{d o}^{H P} \mathcal{D}_{\rho, \omega, 0^{+}}^{\gamma, \mu, \nu} x(t)=A x(t), & t>0, \gamma, \omega \in \mathbb{R}, \rho>0, \\
\mathbf{E}_{\rho,(1-\nu)(1-\mu), \omega, 0^{+}}^{-\gamma(1-\nu)} x(t)_{\left.\right|_{t=0^{+}}}=g_{\rho, \omega}^{\gamma}(\mu, \nu), & 0<\mu<1,0 \leq \nu \leq 1,
\end{aligned}
$$

where $x \in \mathbb{R}^{n}, A \in \mathbb{R}^{n \times n}$ and ${ }_{d o}^{H P} \mathcal{D}_{\rho, \omega, 0^{+}}^{\gamma, \mu, \nu}$ is the distributed order Hilfer-Prabhakar derivative. Then we extend our results to system ${ }_{d o}^{H P} \mathcal{D}_{\rho, \omega, 0^{+}}^{\gamma, \mu, \nu} x(t)=A x(t)+B u(t)$, and at the end of this section we focus on autonomous systems.

In this part we present some results about stability analysis of distributed order HilferPrabhakar linear differential system. We consider the system 3.1 stable if for any initial 
function $g_{\rho, \omega}^{\gamma}$, there would be a $\varepsilon>0$ such that $\|x(t)\|<\varepsilon$ for all $t>0$; moreover, it would be asymptotically stable provided that it is stable and $\|x(t)\| \rightarrow 0$ as $t \rightarrow \infty$.

For introducing the stability theorem for system 3.1, we obtain the Laplace transform of this system. By implementation of the Laplace transform on the system 3.1 and using the initial condition and Lemma 2.8, we have

$$
\begin{aligned}
& \left(1-\omega s^{-\rho}\right)^{\gamma} \int_{0}^{1} \int_{0}^{1} b(\mu, \nu) s^{\mu} d \mu d \nu X(s)- \\
& \quad-\int_{0}^{1} \int_{0}^{1} b(\mu, \nu) s^{-\nu(1-\mu)}\left(1-\omega s^{-\rho}\right)^{\gamma \nu} g_{\rho, \omega}^{\gamma}(\mu, \nu) d \mu d \nu=A X(s),
\end{aligned}
$$

where $\mathcal{L}(x(t))(s)=X(x)$, or

$$
\left(1-\omega s^{-\rho}\right)^{\gamma} B_{1}(s) X(s)-B_{2}(s)=A X(s)
$$

where

and

$$
B_{1}(s)=\int_{0}^{1} \int_{0}^{1} b(\mu, \nu) s^{\mu} d \mu d \nu
$$

$$
B_{2}(s)=\int_{0}^{1} \int_{0}^{1} b(\mu, \nu) s^{-\nu(1-\mu)}\left(1-\omega s^{-\rho}\right)^{\gamma \nu} g_{\rho, \omega}^{\gamma}(\mu, \nu) d \mu d \nu
$$

3.1. Remark. Under the special case 1 of remark 2.11, we have

$$
B_{1}(s)=s^{\mu_{0}},
$$

and

$$
B_{2}(s)=s^{-\nu_{0}\left(1-\mu_{0}\right)}\left(1-\omega s^{-\rho}\right)^{\gamma \nu_{0}} g_{\rho, \omega}^{\gamma}\left(\mu_{0}, \nu_{0}\right)
$$

at this point, if $\nu_{0}=0, \omega=0$ and $\gamma=-1,(3.2)$ will be reduced to the first equation to prove the theorem 3.1 in [38] as

$$
s^{\mu_{0}} X(s)-{ }_{0^{+}} I_{t}^{1-\mu_{0}}(x(t))_{\left.\right|_{t=0^{+}}}=A X(s)
$$

moreover, if $\nu_{0}=1, \omega=0$, and $\gamma=-1$, we will have

$$
s^{\mu_{0}} X(s)-x\left(0^{+}\right)=A X(s) .
$$

In case 2 of remark 2.11, if $b(\mu, \nu)=b(\mu) \delta(\nu-1), \omega=0$, and $\gamma=1$ we will have

$$
B_{1}(s)=\int_{0}^{1} b(\mu) s^{\mu} d \mu
$$

and

$$
B_{2}(s)=\frac{1}{s} \int_{0}^{1} b(\mu) s^{\mu} d \mu x\left(0^{+}\right)
$$

therefore, (3.2) will be reduced to (3.1) in [41].

From remark 3.1, we found that system 3.1 and its stability analysis can be reduced to system 3.1 in $[41,38]$ or system 1 in [32].

Now we recall the final value theorem about Laplace transform.

3.2. Theorem. Let $F(s)$ be the Laplace transform of the function $f(t)$. If all poles of $s F(s)$ are in the open left-half plane, then [21]

$$
\lim _{t \rightarrow \infty} f(t)=\lim _{s \rightarrow 0} s F(s) .
$$


We are now ready to introduce our main theorem based on the asymptotic stability of system 3.1 .

Consider the following assumptions:

H1) All roots of the $\operatorname{det}\left(\left(1-\omega s^{-\rho}\right)^{\gamma} B_{1}(s) I-A\right)=0$ have negative real parts;

H2) $\omega=0$ and $\gamma \leq 1$ or $\omega \neq 0$ and $\gamma \leq \frac{1}{1+\rho}$;

H3) All poles of the $s B_{2}(s)$ are in the open left-half plane.

Based on these, we have the following theorem:

3.3. Theorem. If assumptions $\boldsymbol{H 1}, \boldsymbol{H} 2$ and $\boldsymbol{H} 3$ hold, then the system 3.1 is asymptotically stable.

Proof. From equation 3.2, we have

$$
\left(\left(1-\omega s^{-\rho}\right)^{\gamma} B_{1}(s) I-A\right) X(s)=B_{2}(s) ;
$$

this implies that

$$
\left(\left(1-\omega s^{-\rho}\right)^{\gamma} B_{1}(s) I-A\right) s X(s)=s B_{2}(s) .
$$

From assumption $\mathrm{H} 1$, all roots of the $\operatorname{det}\left(\left(1-\omega s^{-\rho}\right)^{\gamma} B_{1}(s) I-A\right)=0$ lie in open left half of the complex plane (i.e., $\Re(s)<0$ ), so we consider (3.6) in $\Re(s) \geq 0$. In this restricted area, from assumption H1, equation 3.6 has a unique solution $s X(s)=$ $\left(s X_{1}(s), s X_{2}(s), \cdots, s X_{n}(s)\right)^{T}$. But from assumption $\mathrm{H} 2$

$$
\lim _{s \rightarrow 0, \Re(s) \geq 0} s B_{2}(s)=0
$$

thus,

$$
\lim _{s \rightarrow 0, \Re(s) \geq 0} s X_{i}(s)=0, \quad \text { for } i=1,2, \cdots n .
$$

Now by applying the assumption $\mathrm{H} 3$ and final value theorem we will have

$$
\begin{aligned}
& \lim _{t \rightarrow \infty} x(t)=\lim _{t \rightarrow \infty}\left(x_{1}(t), x_{2}(t), \cdots, x_{n}(t)\right)^{T} \\
= & \lim _{s \rightarrow 0, \Re(s) \geq 0}\left(s X_{1}(s), s X_{2}(s), \cdots, s X_{n}(s)\right)^{T}=0 .
\end{aligned}
$$

3.4. Definition. The function $P_{\rho, \omega, A}^{\gamma}(s)=\operatorname{det}\left(\left(1-\omega s^{-\rho}\right)^{\gamma} B(s) I-A\right)$, which is a function in $s$, is called the distributed Hilfer-Prabhakar characteristic function with respect to $B(s)$ of $n \times n$ matrix A, where $B(s)=\int_{0}^{1} \int_{0}^{1} b(\mu, \nu) s^{\mu} d \mu d \nu, b(\mu, \nu) \geq 0$.

It is clear that when $b(\mu, \nu)=b(\mu) \delta(\nu-1)$ and $\omega=0$ and $\gamma=1$, the distributed Hilfer-Prabhakar characteristic function with respect to $B(s)$ of $A$ is the characteristic function of the matrix $A$ defined in [41].

3.5. Definition. The distributed Hilfer-Prabhakar eigenvalues of A(dHPeiv) with respect to $B(s)$ are the roots of the distributed Hilfer-Prabhakar characteristic function of $A$ with respect to $B(s)$.

The inertia of matrix $\mathrm{A}$ of order $\mathrm{n}$, denoted by $\operatorname{In}(A)$, is the triplet $(\pi(A), \nu(A), \delta(A))$, where $\pi(A), \nu(A)$, and $\delta(A)$ are the number of eigenvalues of $A$ with positive, negative, and zero real parts, counting multiplicities (for more details see [18]). Now we generalize the inertia concept for analyzing the stability of distributed order Hilfer-Prabhakar differential systems. 
3.6. Definition. The distributed order Hilfer-Prabhakar inertia of matrix $A$ of order $n$ with respect to $B(s)$, denoted by $\operatorname{In}_{\rho, \omega}^{\gamma}(A)$, is the triplet

$$
\left(\phi_{\rho, \omega}^{\gamma}(A), \chi_{\rho, \omega}^{\gamma}(A), \psi_{\rho, \omega}^{\gamma}(A)\right),
$$

where $\phi_{\rho, \omega}^{\gamma, \mu}(A), \chi_{\rho, \omega}^{\gamma, \mu}(A)$ and $\psi_{\rho, \omega}^{\gamma, \mu}(A)$ are the number of the distributed Hilfer-Prabhakar eigenvalues of $\mathrm{A}$ with respect to $B(s)$ with positive, negative, and zero real parts, counting multiplicities.

It is easy to see that $I n_{B(s)}$, defined in [41], is a special case of $\operatorname{In} n_{\rho, \omega}^{\gamma}(A)$.

3.7. Definition. The matrix $A$ is called a distributed order Hilfer-Prabhakar stable matrix with respect to $B(s)$, if all of the distributed Hilfer-Prabhakar eigenvalues of $A$ with respect to $B(s)$ have negative real parts.

3.8. Theorem. Let assumptions $\boldsymbol{H} 1, H 2$ and $\boldsymbol{H} 3$ hold; then the distributed order HilferPrabhakar differential system 3.1 is asymptotically stable if any of the following equivalent conditions holds.

(1) The matrix $A$ is distributed order Hilfer-Prabhakar stable matrix with respect to $B_{1}(s)$.

(2) $\phi_{\rho, \omega}^{\gamma}(A)=\psi_{\rho, \omega}^{\gamma, \mu}(A)=0$.

(3) All roots of the distributed Hilfer-Prabhakar characteristic function with respect to $B_{1}(s)$ satisfy $|\arg (s)|>\pi / 2$.

Proof. According to definition 3.7, matrix $A$ is a distributed order Hilfer-Prabhakar stable matrix with respect to $B_{1}(s)$, if and only if all distributed Hilfer-Prabhakar eigenvalues of $A$ with respect to $B_{1}(s)$ have negative real parts, that is $\phi_{\rho, \omega}^{\gamma}(A)=\psi_{\rho, \omega}^{\gamma, \mu}(A)=0$. This shows that $(1) \Longleftrightarrow(2)$. Moreover, $\phi_{\rho, \omega}^{\gamma}(A)=\psi_{\rho, \omega}^{\gamma, \mu}(A)=0$, if and only if all distributed Hilfer-Prabhakar eigenvalues of $A$ with respect to $B_{1}(s)$ have negative real parts. This shows that $(2) \Longleftrightarrow(3)$.

Now we consider the following system

$$
\begin{aligned}
& { }_{d o}^{H P} \mathcal{D}_{\rho, \omega, 0^{+}}^{\gamma, \mu, \nu} x(t)=A x(t)+B u(t), \quad t>0, \gamma, \omega \in \mathbb{R}, \rho>0, \\
& \mathbf{E}_{\rho,(1-\nu)(1-\mu), \omega, 0^{+}}^{-\gamma(1-\nu)} x(t)_{\left.\right|_{t=0^{+}}}=g_{\rho, \omega}^{\gamma}(\mu, \nu), \quad 0<\mu<1,0 \leq \nu \leq 1 ;
\end{aligned}
$$

the proof of the following theorem can be easily expressed similar to Theorem 3.3.

3.9. Theorem. If the following assumptions hold, the system 3.9 is asymptotically stable:

a. all roots of the $\operatorname{det}\left(\left(1-\omega s^{-\rho}\right)^{\gamma} B_{1}(s) I-A\right)=0$ have negative real parts;

b. $\lim _{s \rightarrow 0} s\left(B U(s)+B_{2}(s)\right)=0$, where $U(s)=\mathcal{L}(u(x))$;

c. all poles of the $s\left(B U(s)+B_{2}(s)\right)$ are in the open left-half plane.

Next, we will study the stability of the distributed order HilferPrabhakar autonomous systems.

Here, we use linear approximation near a special point-called equilibrium point-of a vector-valued function $F(x)$.

3.10. Definition. The point $\hat{x}$ is an equilibrium point for the autonomous system ${ }_{d o}^{H P} \mathcal{D}_{\rho, \omega, 0^{+}}^{\gamma, \mu, \nu} x(t)=F(x(t))$ if $F(\hat{x})=0$. 
3.11. Definition. An equilibrium solution $\hat{x}$ to an autonomous system of distributed order Hilfer-Prabhakar system is called

a) stable if for every $\epsilon>0$, there exists a $\delta>0$ such that every solution $x(t)$ having initial conditions within distance $\delta>\left\|x\left(t_{0}\right)-\hat{x}\right\|$ of the equilibrium remains within distance $\epsilon>\|x(t)-\hat{x}\|$ for all $t>t_{0}$.

b) asymptotically stable if it is stable and, in addition, there exists $\delta_{0}>0$ such that whenever $\delta>\left\|x\left(t_{0}\right)-\hat{x}\right\|$, then $x(t) \rightarrow \hat{x}$ as $t \rightarrow \infty$.

Equilibrium solutions in which solutions start near them move toward the equilibrium solution are called asymptotically stable equilibrium points or asymptotically stable equilibrium solutions.

Now we consider the distributed order Hilfer-Prabhakar autonomous system as

$$
\begin{aligned}
{ }_{d o}^{H P} \mathcal{D}_{\rho, \omega, 0^{+}}^{\gamma, \mu, \nu} x(t)=F(x(t)), & t>0, \gamma, \omega \in \mathbb{R}, \rho>0, \\
\mathbf{E}_{\rho,(1-\nu)(1-\mu), \omega, 0^{+}}^{-\gamma(1-\nu)} x(t)_{\left.\right|_{t=0^{+}}}=g_{\rho, \omega}^{\gamma}(\mu, \nu), & 0<\mu<1,0 \leq \nu \leq 1,
\end{aligned}
$$

with the equilibrium point $\hat{x}$ that is $F(\hat{x})=0$. Let $x(t)=\hat{x}+r(t)$; then

$$
{ }_{d o}^{H P} \mathcal{D}_{\rho, \omega, 0^{+}}^{\gamma, \mu, \nu} x(t)={ }_{d o}^{H P} \mathcal{D}_{\rho, \omega, 0^{+}}^{\gamma, \mu, \nu} \hat{x}+{ }_{d o}^{H P} \mathcal{D}_{\rho, \omega, 0^{+}}^{\gamma, \mu, \nu} r(t)
$$

also

$$
F(x(t))=F(\hat{x}+r(t))=F(\hat{x})+A r(t)+O(|r(t)|),
$$

where $A$ is the Jacobian matrix of $F(x)$ at the equilibrium point $\hat{x}$, so from (3.11), (3.12) and by using ${ }_{d o}^{H P} \mathcal{D}_{\rho, \omega, 0^{+}}^{\gamma, \mu, \nu} \hat{x}=F(\hat{x})=0$ we have

$$
{ }_{d o}^{H P} \mathcal{D}_{\rho, \omega, 0^{+}}^{\gamma, \mu, \nu} r(t)=A r(t)+O(|r(t)|)
$$

so from (3.13) we have

$$
{ }_{d o}^{H P} \mathcal{D}_{\rho, \omega, 0^{+}}^{\gamma, \mu, \nu} r(t) \simeq A r(t),
$$

with the initial value

$$
\mathbf{E}_{\rho,(1-\nu)(1-\mu), \omega, 0^{+}}^{-\gamma(1-\nu)} r(t)_{\left.\right|_{t=0^{+}}}=g_{\rho, \omega}^{\gamma}(\mu, \nu)-\hat{x} .
$$

Therefore, if $x(t)$ is close to $\hat{x}$, the asymptotic stability of the system

${ }_{d o}^{H P} \mathcal{D}_{\rho, \omega, 0^{+}}^{\gamma, \mu, \nu} r(t)=A r(t)$ with the initial value 3.15 implies that system 3.10 is asymptotically stable at $\hat{x}$.

3.12. Theorem. Let $A$ be the Jacobian matrix of $F(x)$ at the equilibrium point $\hat{x}$ of autonomous system 3.10, and $x(t)$ be close to $\hat{x}$; then the system 3.10 is asymptotically stable at $\hat{x}$ if any of the equivalent conditions in theorem 3.8 holds for Jacobian matrix A.

Proof. From theorem 3.8 and above discussion, the proof of the theorem is obvious.

\section{Examples and Applications}

Now, we illustrate three examples of the systems referred to in the previous section and investigate their stability issues.

4.1. Example. Consider the distributed order Hilfer-Prabhakar system 3.1 with 
$A=\left(\begin{array}{cc}-0.5 & -1 \\ 0.5 & -0.5\end{array}\right)$, under case 1 of remark 2.8 with $\nu_{0}=1$ and $\omega=0$ and initial condition $x(0)=\left(\begin{array}{c}0.1 \\ -0.3\end{array}\right)$. Results of $I n_{\rho, \omega}^{\gamma}(A)$, for $\mu_{0}=0.925,0.825$, and 0.725 are shown in table 1. From table one, we find that the system must be asymptotically stable. From Remark 2.8 and according to [20], we can write the general solution of this system as

$$
x(t)=c_{1} u_{1} E\left(\lambda_{1} t^{\mu_{0}}\right)+c_{2} u_{2} E\left(\lambda_{2} t^{\mu_{0}}\right),
$$

where $c_{1}, c_{2}$ are constants, and $\lambda_{1}, \lambda_{2}$ are eigenvalues, and $u_{1}, u_{2}$ are the corresponding eigenvectors of $A$. Moreover, the asymptotically stability of this system can be seen in figure 1 .

Table 1. Results of the distributed order Hilfer-Prabhakar inertia of matrix $A$ in example 3.9 .

\begin{tabular}{cccc}
\hline$\mu_{0}$ & 0.725 & 0.825 & 0.925 \\
\hline dHPeiv of $A$ & $-0.8135 \pm 0.1031 i$ & $-0.7405 \pm 0.3965 i$ & $-0.6097 \pm 0.6008 i$ \\
\hline In $_{\rho, \omega}^{\gamma}(A)$ & $(0,2,0)$ & $(0,2,0)$ & $(0,2,0)$ \\
\hline
\end{tabular}

4.2. Example. Consider the linearized form of the distributed order fractional WINDMI system at the equilibrium point $\widehat{x}=(\ln b, 0,0)$ as

$$
{ }_{d o}^{C} D_{t}^{\alpha} x(t)=A x(t)
$$

where $x(t)=\left(x_{1}(t), x_{2}(t), x_{3}(t)\right)$ and $A=\left(\begin{array}{ccc}0 & 1 & 0 \\ 0 & 0 & 1 \\ -b & -1 & -a\end{array}\right) ;$ [41] studied the stability analysis of this system. Now we consider the distributed order Hilfer-Prabhakar form of system 4.1 as

$$
\begin{aligned}
{ }_{d o}^{H P} \mathcal{D}_{\rho, \omega, 0^{+}}^{\gamma, \mu, \nu} x(t)=A x(t), & t>0, \gamma, \omega \in \mathbb{R}, \rho>0, \\
\mathbf{E}_{\rho,(1-\nu)(1-\mu), \omega, 0^{+}}^{-\gamma(1-\nu)} x(t)_{\left.\right|_{t=0^{+}}}=g_{\rho, \omega}^{\gamma}(\mu, \nu), & 0<\mu<1,0 \leq \nu \leq 1 .
\end{aligned}
$$

For $b(\mu, \nu)=b(\mu) \delta(\nu-1), \omega=0, \gamma=1$ and $g_{\rho, \omega}^{\gamma}(\mu, \nu)=\widehat{x}$, system 4.2 will be reduced to system 4.1. Now, for analyzing system 4.2 we calculate the distributed order HilferPrabhakar inertia in different cases. To simplify, we set $a=0$ and $b=0$ in case 1 and 2 .

Case 1. Let $b(\mu, \nu)=\delta(\mu-\beta) \delta(\nu-1),(\beta>0), \omega=0, \gamma=1$ and $g_{\rho, \omega}^{\gamma}(\mu, \nu)=\widehat{x}$. In this case, assumption 2 holds and assumption 3 implies that $\beta>0$. Results of $\operatorname{In}_{\rho, \omega}^{\gamma}(A)$ are shown in table 2. We see that the results in table 2 are compatible with results in [41].

Case 2. Let $b(\mu, \nu)=\delta(\mu-\beta) \delta(\nu),(\beta>0), \omega=0, \gamma=-1$ and $g_{\rho, \omega}^{\gamma}(\mu, \nu)=$ $0^{+} I_{t}^{1-\beta}(x(t))_{\left.\right|_{t=0+}}$; these imply that

$$
B_{1}(s)=s^{\beta}, B_{2}(s)=s^{1-\beta} g_{\rho, 0}^{-1}(\beta, 0) .
$$

Therefore, assumption 2 holds and assumption 3 implies that $\beta<2$. Results of calculation of $\operatorname{In}_{\rho, \omega}^{\gamma}(A)$ are shown in table three.

Case 3. Set $b(\mu, \nu)=2 \mu \delta(\nu-1), \omega=0, \gamma=1$ and $g_{\rho, \omega}^{\gamma}(\mu, \nu)=\widehat{x}$. Results for $a$ and $b$ are shown in table 4 . 


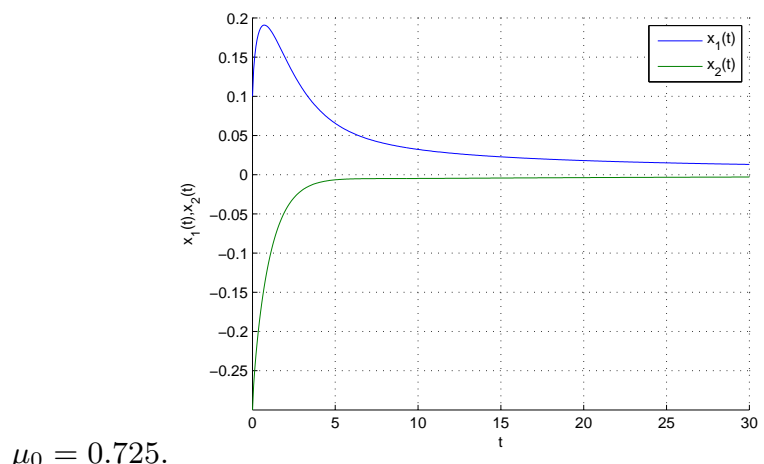

$\mu_{0}=0.725$.

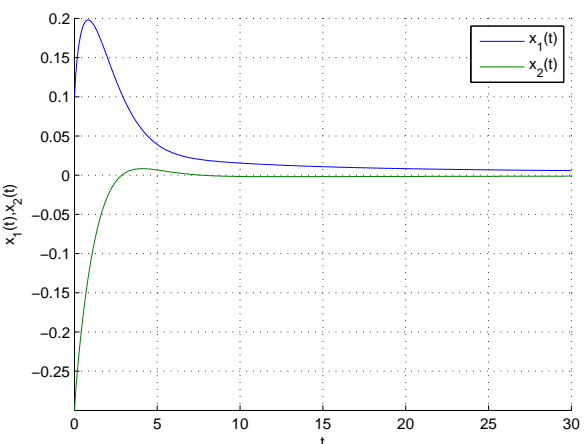

$\mu_{0}=0.825$

$\mu_{0}=0.925$.

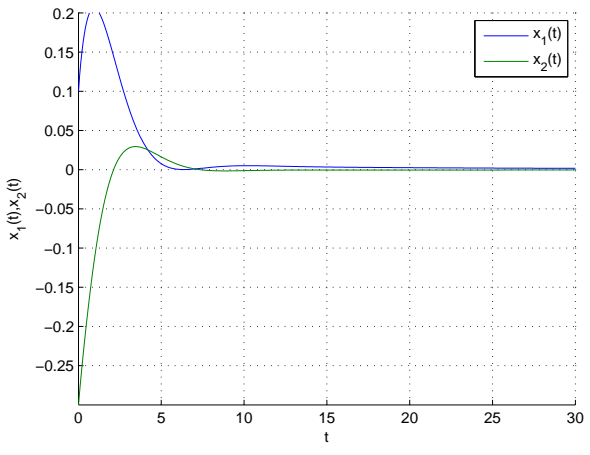

Figure 1. Plot of $x(t)$ in example 3.9 for different $\mu_{0}$.

Table 2. Results of the distributed order Hilfer-Prabhakar inertia of matrix $A$ in example 4.2 case 1.

\begin{tabular}{cccccc}
\hline$\beta$ & 1 & 0.95 & 0.65 & 0.35 & 0.05 \\
\hline $\operatorname{In}_{\rho, \omega}^{\gamma}(A)$ & $(0,0,3)$ & $(0,2,1)$ & $(0,2,1)$ & $(0,0,1)$ & $(0,0,1)$ \\
\hline
\end{tabular}


a)
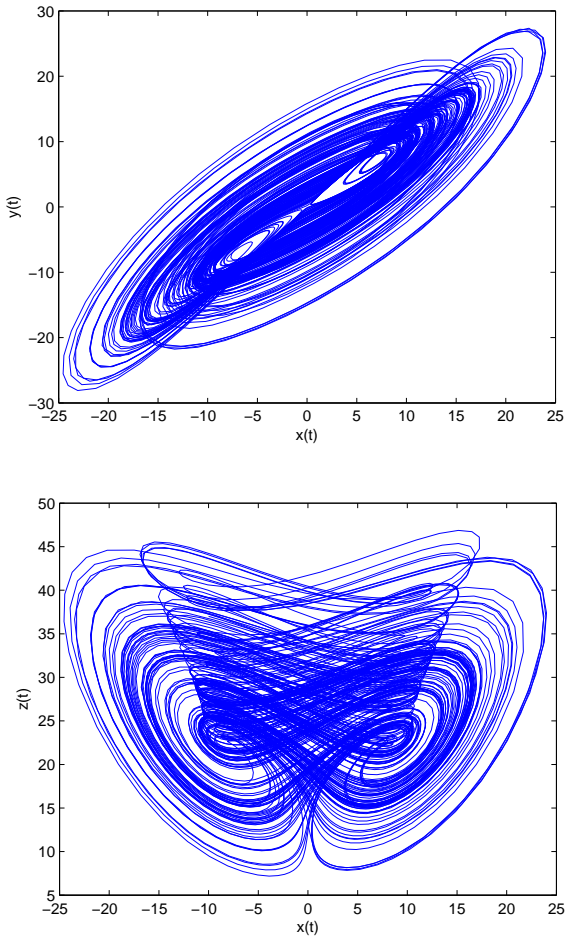

b)

Figure 2. Plot of the integer-order Chen system (case 1) in example 4.3 with $(a, b, c)=(35,3,28)$ in $x y$ and $x z$ plans.

Table 3. Results of the distributed order Hilfer-Prabhakar inertia of matrix $A$ in example 4.2 case 2 .

\begin{tabular}{cccccccccc}
\hline$\beta$ & 1.95 & 1.65 & 1.35 & 1.05 & 1 & 0.95 & 0.65 & 0.35 & 0.05 \\
\hline $\operatorname{In}_{\rho, \omega}^{\gamma}(A)$ & $(2,2,1)$ & $(2,2,1)$ & $(2,0,1)$ & $(2,0,1)$ & $(0,0,3)$ & $(0,2,1)$ & $(0,2,1)$ & $(0,0,1)$ & $(0,0,1)$ \\
\hline
\end{tabular}

From theorem 3.8 and table 2 and 3, we found that the system 3.10 in cases 1 and 2 is not asymptotically stable. Also according to the theorem 3.8 and table 4 we found

Table 4. Results of the distributed order Hilfer-Prabhakar inertia of matrix $A$ in example 4.2 case 3 .

\begin{tabular}{cccccc}
\hline$a$ & 0 & 0 & 1 & 1 & 1 \\
\hline$b$ & 0 & 1 & 1 & 0 & 0.001 \\
\hline$I n_{\rho, \omega}^{\gamma}(A)$ & $(0,1,0)$ & $(0,1,0)$ & $(0,1,0)$ & $(0,1,0)$ & $(0,1,0)$ \\
\hline
\end{tabular}

that the system 4.2 is asymptotically stable in case 3 .

The Lorenz equations were published in 1963 by a meteorologist and mathematician from MIT called Lorenz [42], which are notable for having chaotic solutions for certain 
parameter values and initial conditions. They were derived from a simplified model of convection of the earth's atmosphere. These equations also arise in studies of convection and instability in planetary atmospheres, models of lasers and dynamos, and so forth. In 1999, Chen and Ueta found another simple three-dimensional autonomous system [8], which is not topologically equivalent to Lorenz's system and has a chaotic attractor[8]. As our last example, we will study the distributed order Hilfer-Prabhakar Chen system.

4.3. Example. The Chen system is a system of ordinary differential equations

$$
\begin{gathered}
x^{\prime}(t)=a(y(t)-x(t)), \\
y^{\prime}(t)=(c-a) x(t)-x(t) z(t)+c y(t), \\
z^{\prime}(t)=x(t) y(t)-b z(t),
\end{gathered}
$$

a)
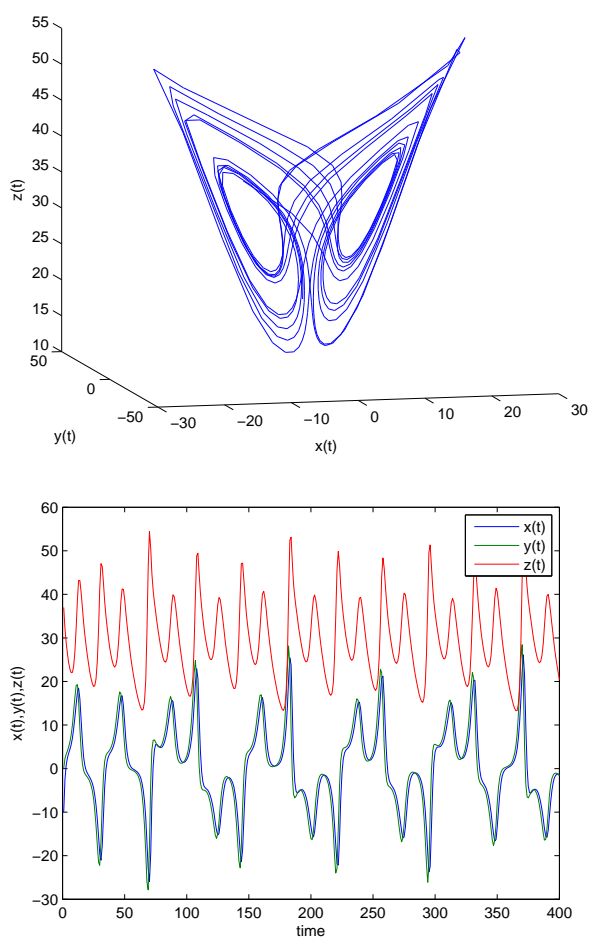

b)

Figure 3. Plot of the system 4.4 (case 2) in example 4.3 with $(a, b, c)=$ $(25,3,28), \mu_{0}=0.9$ and $\mu_{1}=1$.

where $x, y$, and $z$ are the state variables and $a, b$, and $c$ are three system parameters. This system has three equilibrium points $(0,0,0),(\sqrt{c(b-1)}, \sqrt{c(b-1)}, b-1)$ and $(-\sqrt{c(b-1)},-\sqrt{c(b-1)}, b-1)$. The corresponding distributed order Hilfer-Prabhakar of system 4.3 can be written as

$$
\begin{gathered}
{ }_{d o}^{H P} \mathcal{D}_{\rho, \mu, \nu}^{\gamma, \omega, 0^{+}} x(t)=a(y(t)-x(t)), \\
{ }_{d o}^{H P} \mathcal{D}_{\rho, \mu, \nu}^{\gamma, \mu, 0^{+}} y(t)=(c-a) x(t)-x(t) z(t)+c y(t), \\
{ }_{d o}^{H P} \mathcal{D}_{\rho, \omega, 0^{+}}^{\gamma, \mu, \nu} z(t)=x(t) y(t)-b z(t),
\end{gathered}
$$


a)

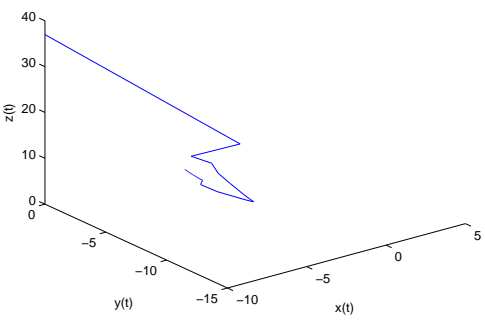

b)

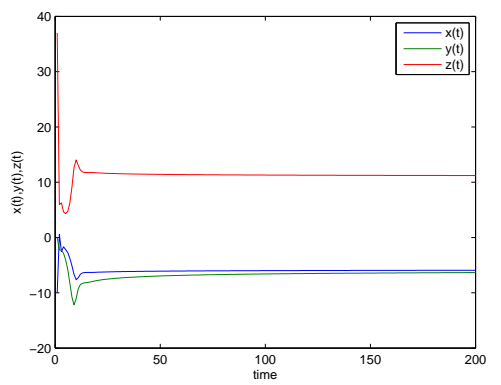

Figure 4. Plot of the system 4.4 (case 2$)$ in example 4.3 with $(a, b, c)=$ $(5,3,8)$ and $\mu_{0}=\mu_{1}=0.5$.

with the initial condition explained in 3.10. The Jacobian matrix of system 4.4 at the equilibrium point $\hat{x}=\left(\hat{x}_{0}, \hat{y}_{0}, \hat{z}_{0}\right)$ is $A=\left(\begin{array}{ccc}-a & a & 0 \\ c-a-\hat{z}_{0} & c & -\hat{x}_{0} \\ \hat{y}_{0} & \hat{x}_{0} & -b\end{array}\right)$. According to the theorem 3.12, system 4.4 is asymptotically stable if matrix $A$ is stable with respect to $B_{1}(s)$. To continue this example we will analyze this system in three different cases with initial condition $(-10,0,37)$ at the equilibrium point $(\sqrt{c(b-1)}, \sqrt{c(b-1)}, b-1)$.

Table 5. Results of the distributed order Hilfer-Prabhakar inertia of matrix $A$ in example 4.3 case 3 .

\begin{tabular}{cccccccc}
\hline$b(\mu, \nu)$ & $\delta(\mu) \delta(\nu-1)$ & \multicolumn{2}{c}{$\delta(\mu-1) \delta(\nu)$} & \multicolumn{2}{c}{$2 \mu \delta(\nu-1)$} & $\nu \sin (\mu)$ \\
\hline$\omega$ & 1 & 1 & 1.5 & 1.5 & 2 & 2 & 2 \\
$\rho$ & 1 & 2 & 1 & 2 & 3 & 1.25 & 1.5 \\
$\gamma$ & 1 & 1 & 1 & 0.5 & 3 & 1.5 & 2.5 \\
$\operatorname{In}_{\rho, \omega}^{\gamma}(A)$ & $(3,0,0)$ & $(3,3,0)$ & $(0,3,0)$ & $(0,3,0)$ & $(11,16,0)$ & $(0,1,0)$ & $(1,0,0)$ \\
\hline
\end{tabular}

Case 1. In case one of remark 2.8 with $\nu_{0}=0, \omega=0$, and $\mu_{0}=1$, system 4.4 reduces to system 4.3. In this case for system 4.4 with $a=35, b=3$, and $c=28$, we have $\operatorname{In}_{\rho, \omega}^{\gamma}(A)=\operatorname{In}(A)=(2,1,0)$. The MATLAB simulations of this system are shown in figure 2. From figure 2 we can see that the system is not asymptotically stable, and so matrix $A$ is not stable.

Case 2. Let $b(\mu, \nu)=\left(\delta\left(\mu-\mu_{0}\right)+\delta\left(\mu-\mu_{1}\right)\right) \delta(\nu-1), \omega=0$, and $\gamma=1$. For $\mu_{0}=0.9$ and $\mu_{1}=1$, the system 4.4 will be reduced to the distributed fractional order Lorenz system with Caputo fractional derivative. In this case the numerical solution has been derived in [8]. The numerical solution for $a=25, b=3$, and $c=28$ are shown in figure 3 and we have $\operatorname{In}_{\rho, \omega}^{\gamma}(A)=(14,16,0)$. Moreover, for $\mu_{0}=\mu_{1}=0.5$ with $a=5, b=3$, and $c=8$, we have $\operatorname{In} n_{\rho, \omega}^{\gamma}(A)=(0,2,0)$, so the system is asymptotically stable. Figure 4 shows this aspect of asymptotic stability.

Case 3. For analyzing the stability of the distributed order Hilfer-Prabhakar Lorenz system, we compute the distributed order Hilfer-Prabhakar inertia of matrix $A$ density function $b(\mu, \nu)$ that varies for parameters $\omega, \rho$, and $\gamma$. The results are shown in table 5 . 


\section{Conclusion}

In this article we discussed the stability analysis of a new class of fractional distributed derivative system which is known as the distributed order Hilfer-Prabhakar derivative and the following results were reached.

1. Generalization of Hilfer-prabhakar and then generalization of concepts such as eigenvalues and inertia for this particular class of fractional distributed derivative. The generalization has been reached in compliance with classical definitions of these concepts.

2. By using the concept of generalized inertia, we investigated the systems stability, and its results are completely listed in theorems $3.3,3.9$ and 3.12 ; that is a generalization of theorems described in [40].

The important point in this article is that without numerical solution or analytical solution and only with the calculation of generalized inertia of a matrix, we were able to evaluate the stability of such systems.

3. And finally to demonstrate the accuracy of the stated claims, which theoretically have been proven, an applicable example that is called Chen system with new derivative, has been studied in this article. We demonstrated this example in three cases.

In case one, by choosing density function $b(\mu, \nu)=\delta(\mu-\beta) \delta(\nu-1)$ and setting $\omega=0$, $\nu_{0}=0$, and $\mu_{0}=1$, the system converts to a system with integer order derivative. In this case, the results of theorem 3.12 was consistent with the simulation results in MATLAB. In the second case, by choosing $b(\mu, \nu)=\left(\delta\left(\mu-\mu_{0}\right)+\delta\left(\mu-\mu_{1}\right)\right) \delta(\nu-1), \omega=0$, and $\gamma=1$. For $\mu_{0}=0.9$ and $\mu_{1}=1$, the results of theorem 3.12 was the same with the numerical results seen in [8].

But in the third case of this example, even though there is no numerical methods for solving this problem, we were able to study the asymptotic stability debate with the help of definition 3.6 and theorem 3.12 .

\section{References}

[1] Aminikhah, H., Refahi Sheikhani, A. and Rezazadeh, H. Travelling wave solutions of nonlinear systems of PDEs by using the functional variable method, Bol. Soc. Paran. Mat., 34 (2), 213-229, 2016.

[2] Aminikhah, H., Refahi Sheikhani, A. H. and Rezazadeh, H. Sub-equation method for the fractional regularized long-wave equations with conformable fractional derivatives, Scientia Iranica, 23 (3), 1048-1054, 2016.

[3] Aminikhah, H., Refahi Sheikhani, A. H. and Rezazadeh, H. Exact solutions of some nonlinear systems of partial differential equations by using the functional variable method, MATHEMATICA, 56 (2), 103-116, 2014.

[4] Aminikhah, H., Refahi Sheikhani, A. H. and Rezazadeh, H. Approximate analytical solutions of distributed order fractional Riccati differential equation, Ain Shams Engineering Journal, Article in press.

[5] Aminikhah, H., Refahi Sheikhani, A. and Rezazadeh, H. Stability analysis of linear distributed order system with multiple time delays, U.P.B. Sci. Bull., 77 (2), 207-218, 2015.

[6] Ansari, A. and Refahi Sheikhani, A. New identities for the Wright and the Mittag-Leffler functions using the Laplace transform, Asian-European Journal of Mathematics, 7 (3), 1-8, 2014.

[7] Ansari, A., Refahi Sheikhani, A. and Kordrostami, S. On the generating function $e^{x t+y \phi(t)}$ and its fractional calculus, Cent. Eur. J. Phys., 11 (10), 1457-1462, 2013.

[8] Aminikhah. H, Refahi Sheikhani, A. and Rezazadeh, H. Stability Analysis of Distributed Order Fractional Chen System, The Scientific World Journal, 2013 (2013), 1-13, 2013.

[9] Ansari, A., Refahi Sheikhani, A. and Saberi Najafi, H. Solution to system of partial fractional differential equations using the fractional exponential operators, Math. Meth. Appl. Sci., 35 (1), 119-123, 2012. 
[10] Atanackovic,T.M. A generalized model for the uniaxial isothermal deformation of a viscoelastic body, Acta Mech., 159 (1), 77-86, 2002.

[11] Atanackovic, T.M., Budincevic, M. and Pilipovic, S. On a fractional distributed-order oscillator, J. Phys. A, Math. Gen., 38 (30), 6703-6713, 2005.

[12] Atanackovic, T.M., Pilipovic, S. and Zorica, D. Time distributed order diffusion-wave equation, II. Applications of the Laplace and Fourier transformations, Proc. R. Soc. A, 465 (2106), 1893-1917, 2009.

[13] Atanackovic, T.M., Pilipovic, S. and Zorica, D. Distributed-order fractional wave equation on a finite domain. Stress relaxation in a rod, Int. J. Eng. Sci., 49 (2), 175-190, 2011.

[14] Bagley, R. L. and Torvik, P.J. On the existence of the order domain and the solution of distributed order equations, International Journal of Applied Mathematics, 2 (8), 965-987, 2000 .

[15] Blasius. B., Huppert, A. and Stone, L. Complex dynamics and phase synchronization in spatially extended ecological systems, Nature, 399 (6734), 354-359, 1999.

[16] Caputo, M. Mean fractional-order-derivatives differential equations and filters, Annali dell'Università di Ferrara. Nuova Serie. Sezione VII. Scienze Matematiche, 41 (1), 73-84, 1995.

[17] Caputo, M. Distributed order differential equations modelling dielectric induction and diffusion, Fractional Calculus Applied Analysis, 4 (4), 421-442, 2001.

[18] Datta, B. N. Stability and inertia, Linear Algebra and its Application, 302, 563-600, 1999.

[19] Denisov, V.I., Shvilkin, B.N., Sokolov, V.A. and Vasili'Ev, M.I. Pulsar radiation in postMaxwellian vacuum nonlinear electrodynamics, Physical Review D - Particles, Fields, Gravitation and Cosmology, 94 (4), 1-6, 2016.

[20] Diethelm, K. The Analysis of Fractional Differential Equations: An Application-Oriented Exposition Using Differential Operators of Caputo Type, vol. 2004 of Lecture Notes in Mathematics, Springer, Berlin, Germany, 2010.

[21] Duffy, D. G. Transform Methods for Solving Partial Differential Equations, CRC Press, 2nd edition, 2004.

[22] Garra, R., Gorenflo, R., Polito, F. and Tomovski, Z. Hilfer-Prabhakar derivatives and some applications, Applied Mathematics and Computation, 242, 576-589, 2014.

[23] Garrappa, R. GrnwaldLetnikov operators for fractional relaxation in HavriliakNegami models, Communications in Nonlinear Science and Numerical Simulation, 38, 178-191, 2016.

[24] Gorenflo, R., Kilbas, A. A., Mainardi, F. and Rogosin, S. V. Mittag-Leffler functions, related topics and applications, Berlin: Springer, 2014.

[25] Han, S. K., Kurrer, C. and Kuramoto, Y. Dephasing and bursting in coupled neural oscillators, Phys. Rev. Lett., 75 (17), 3190-3193, 1995.

[26] Hilfer, R. Applications of fractional calculus in physics, World Scientific, Singapore, 2000.

[27] Hilfer, R. Experimental evidence for fractional time evolution in glass forming materials, Chem. Phys., 284, 399408, 2002.

[28] Humbert, P. Quelques resultats d'le fonction de Mittag-Leffler, C. R. Acad. Sci. Paris, 236 (1953), 1467-1468, 1953.

[29] Kilbas, A. A., Saigo, M. and Saxena, R.K. Generalized MittagLeffler function and generalized fractional calculus operators, Integr. Transf. Spec. Funct., 15 (1), 31-49, 2010.

[30] Kilbas, A. A., Srivastava, H.M. and Trujillo, J.J. Theory and Applications of Fractional Differential Equations, in: North-Holland Mathematical Studies, vol. 204, Elsevier (NorthHolland) Science Publishers, Amsterdam, 2006.

[31] Lakshmanan, M. and Murali, k. Chaos in Nonlinear Oscillators: Controlling and Synchronization,World Scientific Series on Nonlinear Science Series A: 13, 1996.

[32] Matignon, D. Stability results for fractional differential equations with applications to control processing, Computational Engineering in Systems and Application Multiconference, IMACS, IEEE-SMC, Lille, France, 2 (1996), 963-968, 1996.

[33] Mittag-Leffler, G. Sur la nouvelle fonction $E_{\alpha}(x)$, Comptes Rendus de 1Academie des Sciences Paris, 137, 554-558, 1903.

[34] Mittag-Leffler, G. Sur la representation analytique d'une branche uniforme d'une fonction monogene (cinquieme note), Acta Math., 29, 101-181, 1905. 
[35] Polito, F. and Scalas, E. A generalization of the space-fractional Poisson process and its connection to some Lévy processes, Electronic Communications in Probability, 21, 1-14, 2016.

[36] Polito, F.and Tomovski, Z. Some properties of Prabhakar-type fractional calculus operators, Fractional Differ. Calc, 6 (1), 73-94, 2016.

[37] Prabhakar, T. R. A singular integral equation with a generalized Mittag-Leffler function in the kernel, Yokohama Math. J., 19 (1971), 7-15, 1971.

[38] Qian, D., Li, C., Agarwal, R. P. and Wong, P. J. Y. Stability analysis of fractional differential system with Riemann-Liouville derivative, Mathematical and Computer Modelling, 52 (56), 862-874, 2010.

[39] Refahi Sheikhani, A., Saberi Najafi, H., Ansari, A,. and Mehrdoust, F. Analytic study on linear system of distributed order fractional differential equation,Le Matematiche, 67 (2), 3-13, 2012.

[40] Rezazadeh, H., Aminikhah, H. and Refahi Sheikhani, A. Stability analysis of Hilfer fractional differential systems, Math. Commun, 21 (1), 45-64, 2016.

[41] Saberi Najafi, H., Refahi Sheikhani, A. and Ansari, A. Stability Analysis of Distributed Order Fractional Differential Equations, Abstract and Applied Analysis., 2011 (2011), 112, 2011.

[42] Sparrow, C. The Lorenz Equations: Bifurcations, Chaos, and Strange Attractors, Springer, New York, NY, USA, 1982.

[43] Srivastava, H. M. and Zhukovsky, K. V. Solutions of Some Types of Differential Equations and of Their Associated Physical Problems by Means of Inverse Differential Operators, Th. M. Rassias and V. Gupta (Editors), In book: Mathematical Analysis, Approximation Theory and Their Applications, Springer optimization and its applications series, 573-629, 2016.

[44] Wiman, A. ber de Fundamental Satz in der Theorie der Funktionen $E_{\alpha}(x)$, Acta Math., 29 (1), 191-201. 1905.

[45] Yu, Y., Li, H., Sha Wang, S. and Yu, J. Dynamic analysis of a fractional-order Lorenz chaotic system, Chaos, Solitons and Fractals, 42 (2), 1181-1189, 2009.

[46] Zhukovsky, K. V. Exact solution of Guyer-Krumhansl type heat equation by operational method, International Journal of Heat and Mass Transfer, 96, 132-144, 2016.

[47] Zhukovsky, K. V. Violation of the maximum principle and negative solutions with pulse propagation in Guyer- Krumhansl model, International Journal of Heat and Mass Transfer, 98, 523-529, 2016 .

[48] Zhukovsky, K. V. A method of inverse differential operators using ortogonal polynomials and special functions for solving some types of differential equations and physical problems, Moscow Univ. Phys. Bull., 70 (2), 93-100, 2015.

[49] Zhukovsky, K. V. The Operational Solution of Fractional-Order Differential Equations, as well as BlackScholes and Heat-Conduction Equations, Moscow Univ. Phys. Bull., 71 (3), 237-244, 2016.

[50] Zhukovsky, K.V. Operational method of solution of linear non-integer ordinary and partial differential equations, SpringerPlus, 5 (1), 1-25, 2016.

[51] Zhukovsky, K. V. and Srivastava, H. M. Analytical solutions for heat diffusion beyond Fourier law, Applied Mathematics and Computation, 293, 423-437, 2017.

[52] Zhukovsky, K.V. Operational solution for some types of second order differential equations and for relevant physical problems, Journal of Mathematical Analysis and Applications, 446 (1), 628-647, 2017. 